\title{
Traqueostomia percutânea: técnica ideal?
}

\section{Percutaneous Tracheosthomy: ideal technique?}

\author{
Rodrigo Afonso da Silva Sardenberg ${ }^{1}$; Antonio Bommim M. Avertano'; Riad N. Younes²
}

RE S U M O

\begin{abstract}
A traqueostomia percutânea tem substituído a traqueostomia convencional em muitas Unidades de Terapia Intensiva, devido à facilidade e segurança do método realizado à beira do leito, dispensando o transporte do paciente da Unidade de Terapia Intensiva para o Centro Cirúrgico. Alguns autores sugerem que a dissecção limitada resulta em menor dano tecidual, diminui o risco de sangramento e infecção de ferida operatória. Esta revisão visa analisar e sintetizar estudos prospectivos e randomizados, revisões sistemáticas e meta-análises, comparando a traqueostomia percutânea e traqueostomia convencional em pacientes adultos em Unidades de Terapia Intensiva.
\end{abstract}

Descritores: Pacientes. Procedimentos cirúrgicos operatórios. Respiração artificial. Traqueostomia. Técnicas.

\section{INTRODUÇÃO}

T raqueostomia é um dos procedimentos mais realizados em pacientes críticos, especialmente nas Unidades de Terapia Intensiva, e com previsão de aumento devido à demanda crescente. A indicação precoce nos pacientes que necessitam de ventilação mecânica prolongada, comparada à intubação translaríngea, tem sido extensamente discutida, ainda que o melhor momento para sua realização é objeto de discussão.

Tal procedimento é um dos mais antigos da medicina, sendo descrito até mesmo em livros de medicina da antiguidade. Galeno, renomado médico grego na época, realizou traqueostomia para tratamento de obstrução de via aérea superior no Século $\| \mathrm{aC}$. A primeira traqueostomia realizada com sucesso, no entanto, é creditada a Antonio Brasalova, em 1546, médico italiano, em um paciente com abscesso laríngeo'.

A técnica cirúrgica atual foi desenvolvida por Jackson ${ }^{1}$ há aproximadamente 100 anos, e, desde então, tem sofrido poucas modificações e refinamentos, sendo que a morbidade e mortalidade relacionadas ao método têm baixo índice. Uma nova técnica, semelhante à traqueostomia percutânea através de canulação traqueal às cegas, iniciou-se, em 1955, com Shelden et al. ${ }^{1}$, porém devido à alguns acidentes, inclusive fatais, esta técnica não obteve boa aceitação. Em 1969, Toye e Weinstein² desenvolveram guia e dilatador com o intuito de facilitar a passagem da cânula.

A técnica de traqueostomia percutânea foi primeiramente realizada por $\mathrm{Ciaglia}^{3}$, e incluindo suas modi- ficações, tornou-se a mais difundida e utilizada forma de traqueostomia.

Estima-se atualmente que aproximadamente 2/ 3 das traqueostomias sejam realizadas desta forma nas Unidades de Terapia Intensiva (UTI).

Na primeira parte desta revisão consideraremos as várias técnicas de traqueostomia percutânea. Na segunda, abordaremos revisões que comparam as técnicas de traqueostomia percutânea (TP) e traqueostomia convencional (TC), proporcionando uma visão crítica de ambos os métodos, assim como complicações, indicações e contraindicações relacionadas a cada uma.

\section{MÉTODOS}

Foram incluídos nesta revisão publicações acerca deste assunto, de janeiro de 1990 até maio de 2008, com as palavras traqueostomia percutânea, traqueostomia cirúrgica ou convencional e traqueostomia na UTI, extraídas da literatura, em inglês, a partir do MEDLINE (Pubmed). Estão descritos estudos prospectivos randomizados, séries de casos, casos clínicos, revisões sistemáticas e metanálises.

Uma iniciativa para a coleta de dados foi conduzida com o intuito de se buscar estudos que realizaram comparações entre os métodos de traqueostomias. Neste sentido, análises das diferentes técnicas de traqueostomias e entre TP e TC, foram condições essenciais para a realização desta revisão crítica.

Várias técnicas de TP e complicações relacionadas a elas foram descritas ${ }^{9}$. Da mesma forma que a TC,

Trabalho realizado no Departamento de Cirurgia Torácica do Hospital AC Camargo- São Paulo - SP-BR.

1. Cirurgião Torácico do Hospital AC Camargo - São Paulo - SP-BR; 2. Chefe Departamento Cirurgia Torácica do Hospital AC Camargo- São Paulo - SP-BR. 
todo preparo deve ser providenciado: sedação adequada, extensão cervical, ventilação mecânica ajustada (PEEP $<5 \mathrm{~cm}$ $\mathrm{H}_{2} \mathrm{O}$ e $\mathrm{FIO} 2=100 \%$ ) e monitorização contínua.

A punção deverá ser realizada preferencialmente entre o segundo e terceiro anéis traqueais. Há evidências de que a punção mais distal está associada ao menor dano de vasos de maior calibre, cordas vocais ou quaisquer outras variações anatômicas.

O método de Ciaglia ${ }^{3}$, descrito em 1985, utiliza dilatadores progressivos, até que o estoma tenha tamanho suficiente para a introdução da cânula. Desde que foi relatada, esta técnica sofreu basicamente três modificações: abertura traqueal entre anéis mais distal em relação à cartilagem cricoide; uso rotineiro de broncoscopia (sempre que possível); os dilatadores progressivos foram substituídos por dilatadores (Blue Rhino-Cook) ou pinça (Griggs) únicos.

Na técnica descrita por Griggs et al. ${ }^{4}$, em 1990, utilizou-se pinça especialmente desenhada, com orifício para introdução do fio guia (Figura 1). A punção traqueal é realizada preferencialmente orientada por broncoscopia e a pinça de Griggs (KIT da Portex), então é passada pelo fio guia através do canal. Esta pinça permite abertura até que a dilatação traqueal seja o suficiente para a introdução da cânula.

Os vários dilatadores progressivos foram substituídos por dilatador único curvo flexível (Figura 2), o qual permite completa dilatação em uma única etapa. Após punção de Seldinger ${ }^{5}$, tal dilatador é introduzido por um cateter até a marca de segurança (38F). A introdução e dilatação poderão ser feitas com movimentos suaves, devido à natureza do material.

A traqueostomia translaríngea ou técnica de Fantoni ${ }^{6}$, consiste na dilatação retrógrada guiada por broncoscopia. Neste método, uma cânula com formato de cone e ponta de metal é introduzida pela luz traqueal, perfurando e dilatando-a em direção à pele.

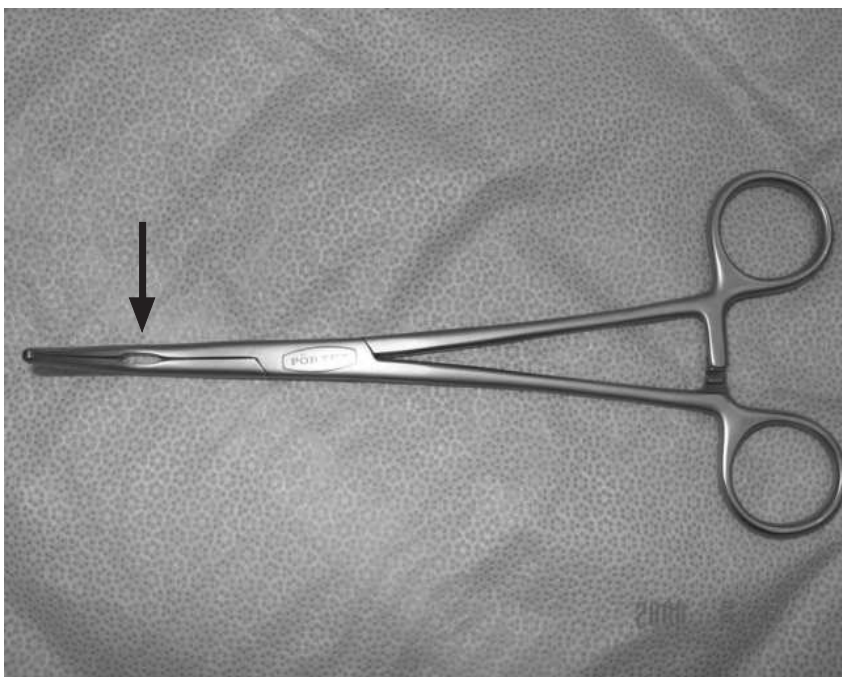

Figura 1 - $\quad$ Pinça de Griggs com canal interno para passagem de fio guia.
Em 2002, Frova e Quintel7 descreveram uma nova técnica que utiliza um dilatador único (PercuTwistRusch), que pode ser introduzido pelo estoma traqueal por movimento de rotação (parafuso). Resultados preliminares não mostraram complicações maiores.

\section{DISCUSSÃO}

Vários estudos ${ }^{8-11}$ avaliaram as técnicas incluindo as referentes à utilização do Blue Rhino (Cook) ou Ciaglia e Pinça de Griggs (Portex). Muito embora existam divergências, não há, na literatura médica, informações consistentes quanto à melhor técnica.

Outra questão importante referente aos pacientes de UTI, diz respeito à escolha do método: traqueostomia percutânea ou traqueostomia convencional?

Traqueostomia Percutânea: técnica ideal?

Revisão sistemática e metanálise realizada por Delaney et al. ${ }^{8}$, analisou 1212 pacientes submetidos à traqueostomia (TC x TP). Na maioria (71\%) das TP foram utilizados vários dilatadores e $94 \%$ desses procedimentos foram realizados na UTI. O índice de infecção no sítio operatório foi menor na TP (OR 0.28, IC 95\%: 0.16-0.49). Porém, de maneira geral, para sangramentos, outras complicações peri e pós-operatórias e óbito, as cifras foram equi-

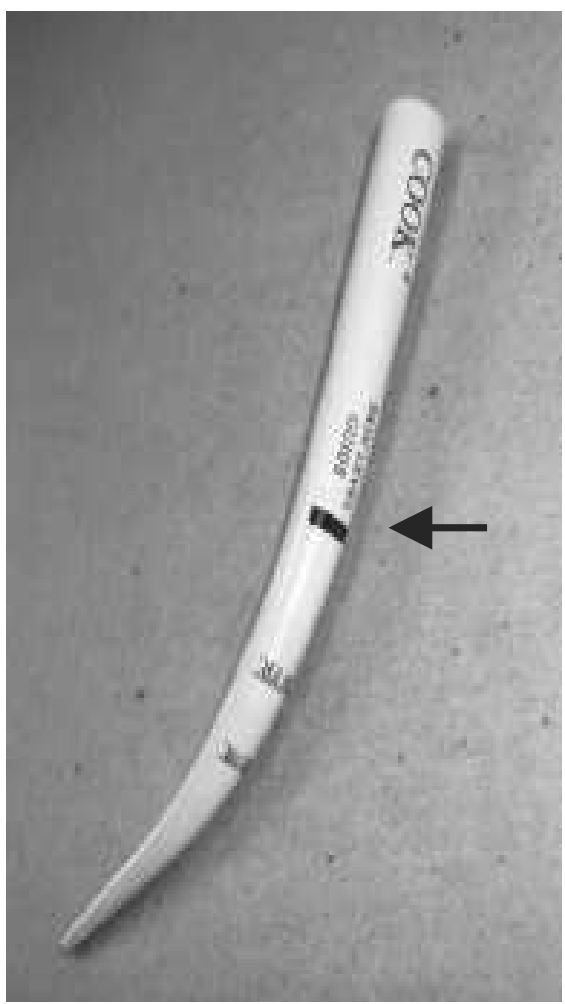

Figura 2 - Dilatador Blue Rhino. O tamanho do estoma pode ser controlado limitando-se a profundidade de sua inserção pela linha de segurança (seta). 
valentes para TP e TC. Existe, no entanto, uma tendência menor de sangramento precoce, tardio e mortalidade na TP. Os autores concluem dessa forma, que a TP deve ser a técnica de escolha para a realização da traqueostomia eletiva.

Outra revisão clínica feita por Al-Ansari et al. ${ }^{9}$, analisou 532 pacientes em oito estudos prospectivos randomizados e controlados, e verificou menor incidência de complicações com a TP, e concluíram que esta técnica é segura.

Melloni et al..$^{10}$, assim como Freeman et al. ${ }^{11}$, descreveram em estudo prospectivo e randomizado, com seguimento em longo prazo, que a TP é um método mais simples e rápido do que a TC. Por outro lado, as complicações tardias foram maiores com esta técnica, porém sem impacto estatístico.

Kearney et al. ${ }^{12}$ publicaram estudo com $827 \mathrm{pa}$ cientes submetidos à TP, e mostraram complicações intraoperatórias menores, ao redor de 6\%, e pós-operatórias de $5 \%$.

Interessante é o fato de que, embora não existam divergências no momento da indicação da traqueostomia (TP x TC), mudanças neste sentido ocorreram. Atualmente, a TP está sendo indicada mais precocemente do que a TC, em torno de 4,5 dias. Isto pode ser explicado provavelmente por: diminuição da disponibilidade de salas cirúrgicas; preocupação em transportar o paciente grave da UTI e maior conforto em realizar a TP na própria UTI. Somente dois estudos ${ }^{9,11}$ compararam este momento da decisão, e ambos encontraram tempo significativamente mais curto com a TP. Além disto, este método ofereceu benefícios adicionais aos pacientes graves, como diminuição da sedação, desmame mais precoce e menor tempo de permanência na UTI.

Quando comparado ao método descrito inicialmente por Ciaglia3 ${ }^{3}$ a utilização do "kit Blue-Rhino" apresentou menor índice de complicações graves. De acordo com estudo realizado por Byhahn et al. ${ }^{13}$, ocorreram duas lesões em parede posterior e um pneumotórax, tais lesões foram atribuídas à inexperiência da equipe com o método. Estima-se que o tempo para a realização da TP com BlueRhino -três minutos em média- é comparável com o tempo gasto com a pinça de Griggs, ou seja, aproximadamente dois minutos.

Estudo prospectivo e randomizado ${ }^{14}$, em pacientes vítimas de trauma, o uso de dilatador único foi comparado com dilatadores múltiplos, e mostrou-se superior devido a menor perda de volume corrente durante o procedimento.

Uma das principais vantagens está associada à facilidade da realização à beira do leito, evitando o risco potencial do transporte de pacientes graves para o Centro Cirúrgico. A TP realizada em UTI é procedimento menos dispendioso quando comparado à TC realizada no Centro Cirúrgico (US\$.1569 p/TP x US\$.3172 p/TC). Conforme já discutido anteriormente, as infecções de ferida operatória e sangramentos também são menores com a TP. Como a dissecção de tecidos é menor, a cânula se acomoda melhor.

Assim como ocorre com qualquer outra técnica cirúrgica nova, o entusiasmo introduziu e incrementou a utilização desta técnica ao longo dos anos, tornando a TP responsável por $60 \%$ de todas as traqueostomias realizadas.

\section{Uso da Broncoscopia}

Embora não haja estudos randomizados e controlados mostrando o benefício real da broncoscopia, este aparato permite confirmação da punção, dilatação, introdução e ajuste da cânula, além da aspiração de secreções ou coágulos. Se por um lado nenhum estudo mostrou que a broncoscopia é indispensável, por outro, há despreocupação da equipe com efeitos indesejáveis quando esta é utilizada $^{15}$. Aumento na pressão intracraniana pode ser observada devido ao aumento da tensão do $\mathrm{CO}_{2}$, além de vazamento de gás e extubação acidental.

A capnografia vem sendo utilizada como potencial alternativa á broncoscopia. Mallick et al. ${ }^{16}$ utilizaram este método em 26 pacientes, e broncoscopia em 29. Confirmaram que o tempo de procedimento, complicações perioperatórias e eficácia do método foram similares em ambos os grupos.

Paran et al. ${ }^{15}$ relataram que uma técnica de TP modificada, na qual há dissecção dos tecidos pré-traqueais, é segura o suficiente sem a utilização da broncoscopia.

Apesar de controverso, a utilização rotineira da broncoscopia na TP ainda é empregada ${ }^{15}$, devido à sua disponibilidade, baixo custo e segurança.

\section{Situações Especiais}

As situações citadas a seguir podem ser consideradas contraindicações relativas ou absolutas para a realização da TP, porém a experiência da equipe é o fator decisivo para a escolha da melhor técnica.

A traqueostomia de urgência, inicialmente considerada como contra-indicação absoluta para TP, atualmente já se advoga a sua realização com segurança para obtenção de via aérea de urgência. Segundo Klein et al. ${ }^{14}$, este procedimento pode ser realizado em situações de emergência, desde que por equipes experientes.

Os pacientes com obesidade mórbida submetidos à TP apresentam um risco 2,7 maior de complicações peri-operatórias e 4,9 maior de morbidades graves ${ }^{13}$. Para estes pacientes recomenda-se a dissecção romba dos tecidos pré-traqueais, permitindo então a palpação traqueal. Uma vez optando-se pela TP, é importante que o pescoço esteja hiperextendido para que haja palpação de algum ponto de reparo.

A presença do bócio tireoidiano pode dificultar a palpação e identificação das estruturas cervicais, mas não constitui contra-indicação ao método. A extensão cervical adequada, a punção ao nível do primeiro anel 
traqueal, ou o auxílio de ultrassom cervical, tornam a TP possível. Vale lembrar que mesmo realizada transtireoide, a dissecção é mínima e o sangramento normalmente é autolimitado ${ }^{8,15}$.

A incapacidade para a hiperextensão cervical é considerada contraindicação para realização da TP. Em pósoperatório de artrodese de coluna cervical, considera-se seguro a confecção da TP a partir do sétimo dia de pósoperatório ${ }^{10,11}$. A utilização de USG cervical utilizado como guia à TP poderá ser útil. Estudo ${ }^{9}$ mostrou $96 \%$ de sucesso e 7,1\% de complicações em pacientes que não tinham possibilidade de extensão cervical adequada.

Historicamente, a presença de traqueostomia prévia é contraindicação para realização de TP. Por outro lado, a presença de cicatriz prévia de certa forma direciona a punção e possivelmente há menos tecidos pré-traqueais, o que facilitaria o procedimento. Meyer e al. ${ }^{17}$ reportaram casuística em 14 pacientes com traqueostomia prévia, onde a TP foi realizada com sucesso.

Pacientes com insuficiência respiratória grave, com status ventilatório muito desfavorável (PEEP>10 $\mathrm{mmHg}$, FlO2>70\%), tradicionalmente são submetidos à TC. Tal fato é explicado pela possível hipoxemia que pode ocorrer durante a manipulação com o broncoscópio, na qual o tubo orotraqueal é tracionado. Há relato ${ }^{11}$ de realização de TP sem maiores problemas nestas condições.
A primeira medida a ser tomada antes da traqueostomia é corrigir as coagulopatias. Normalmente estes pacientes são submetidos à TC no centro cirúrgico. Auzinger et al. ${ }^{18}$ publicaram importante estudo prospectivo em pacientes com coagulopatia e hepatopatia graves. Neste estudo, 60 pacientes foram submetidos à TP na UTI, e apenas um paciente apresentou sangramento importante que cessou após a inserção da cânula. A experiência da equipe médica e a correção das discrasias sanguíneas são fatores determinantes do sucesso.

Para a exérese de volumosos tumores cervicais, geralmente o cirurgião de cabeça e pescoço está envolvido, e, muitas vezes, durante o procedimento para tratamento do tumor primário, a traqueostomia se faz necessária. Quando há obstrução de via aérea por tumor cervical, é imprescindível o bom senso, ou seja, consultar o especialista na área e optar pela técnica mais segura ${ }^{14}$.

Consideramos que a traqueostomia percutânea é um procedimento que pode ser realizado à beira do leito com baixa morbidade, por equipes experientes, mesmo nos pacientes com coagulopatia importante, pois a ocorrência de sangramento grave é infrequente. Atualmente esta técnica é recomendada como o procedimento de escolha para os pacientes de UTI, e, sempre que possível, orientada por broncoscopia.

\title{
A B S T R A C T
}

\begin{abstract}
Objectives: The percutaneous dilatational tracheostomy has become the technique of choice in the Intensive Care Unit, due safety and facilities to be performed at bedside, wich may overcome the risks associated with transport of critically ill patients to the Operating Room. Proponents of percutaneous dilatational tracheostomy suggest that the limited dissection results in less tissue damage, lowers the risk of bleeding and wound infection. The aim of this study is to review the techniques of percutaneous dilatational tracheostomy through a critical analysis of it's indications, contra-indications and complications, as well to compare percutaneous dilatational tracheostomy to surgical tracheostomy. This manuscript analysis and synthesizes randomized prospective studies, meta-analysis and systematic reviews, comparing both techniques in adult critically ill patients in the Intensive Care Unit.
\end{abstract}

Key words: Patients. Surgical procedures, operative. Respiration, artificial. Tracheostomy. Techniques.

\section{REFERENCIAS}

1. Shelden $\mathrm{CH}$, Pudenz RH, Tichy FY. Percutaneous tracheothomy. J Am Med Assoc 1957;165(16):2068-70.

2. Toye FJ, Weinstein JD. Clinical experience with percutaneous tracheostomy and cricothyroidotomy in 100 patients. J Trauma 1986;26(11):1034-40.

3. Ciaglia P, Firsching R, Syniec $C$. Elective percutaneous dilatational tracheostomy. A new simple bedside procedure: preliminary report. Chest 1985;87(6):715-9

4. Griggs WM, Worthley LI, Gilligan JE, Thomas PD, Myburg JA. A simple percutaneous tracheostomy technique. Surg Gynecol Obstet 1990;170(6):543-5

5. Seldinger SI. Catheter replacement of the needle in percutaneous arteriography; a new technique. Acta radiol 1953;39 (5):368-76.

6. Fantoni A. Translaryngeal tracheostomy. In: Gullo A, editor. Anaesthesia, Pain, Intensive Care and Emergency. Triete: APICE; 1993. p.459-65.
7. Frova G, Quintel M. A new simple method for percutaneous tracheostomy: controlled rotating dilation. A preliminary report. Intensive Care Med 2002;28(3):299-303.

8. Delaney A, Bagshaw SM, Nalos M. Percutaneous dilatational tracheostomy versus surgical tracheostomy in crtically ill patients: a systematic review and meta-analysis. Crit Care 2006;10(2):R55.

9. Al-Ansari MA, Hijazi MH. Clinical review: percutaneous dilatational tracheostomy. Crit Care 2006;10(1):202.

10. Melloni G, Muttini S, Gallioli G, Carretta A, Cozzi S, Gemma M, et al. Surgical tracheostomy versus percutaneous dilatational tracheostomy. A prospective-randomized study with long-term follow-up. J Cardiovasc Surg 2002;43(1):113-21.

11. Freeman BD, Isabella K, Lin N, Buchman TG. A meta-analysis of prospective trials comparing percutaneous and surgical tracheostomy in critically ill patients. Chest 2000; 118(5):1412-8.

12. Johnson KL, Kearney BD, Johnson SB, Niblett JB, MacMillan NL, $\mathrm{McClain} \mathrm{RE}$. Closed versus open endotracheal suctioning: costs and physiologic consequences. Crit Care Med 1994;22(4):658-66. 
13. Byhahn C, Wilke HJ, Halbig S, Lischke V, Westphal K. Percutaneous tracheostomy: ciaglia blue rhino versus the basic ciaglia technique of percutaneous dilatational tracheostomy. Anesth Analg 2000;91(4):882-6.

14. Mallick A, Quinn AC, Bodenham AR, Vucevic M. Use of the Combitube for airway maintenance during percutaneous dilatational tracheostomy. Anaesthesia 1998;53(3): 249-55.

15. Paran H, Butnaru G, Hass I, Afanasyv A, Gutman M. Evaluation of a modified percutaneous tracheostomy technique without bronchoscopic guidance. 2004;126(3): 868-71.

16. Klein M, Weksler N, Kaplan DM, Weksler D, Chorny I, Gurman GM. Emergency percutaneous tracheostomy is feasable in experienced hands. Eur J Emerg Med 2004; 11(2):108-12.

17. Meyer M, Critchlow J, Mansharamani N, Angel LF, Garland R, Ernst A. Repeat bedside percutaneous dilational tracheostomy is a safe procedure. Crit Care Med 2002;30(5): 986-8.

18. Auzinger G, O'Callaghan GP, Bernal W, Sizer E, Wendon JA. Percutaneous tracheostomy in patients with severe liver disease and a high incidence of refractory coagulopathy: a prospective trial. Crit Care 2007;11(5):R110.
Recebido em: 18/07/2010

Aceito para publicação em: 20/09/2010

Conflito de interesse: nenhum

Fonte de financiamento: nenhum

\section{Como citar este artigo:}

Silva RA, Avertano ABM, Younes RN. Traqueostomia percutânea: téc nica ideal ? Rev Col Bras Cir. [periódico na Internet] 2011; 38(6). Disponível em URL: http://www.scielo.br/rcbc

\section{Endereço para correspondência:}

Rodrigo Afonso da Silva

E-mail: rodafs@uol.com.br 